\title{
Menopausia y riesgo cardiovascular
}

\author{
EUGENIO ARTEAGA URZÚA
}

Departamento de Endocrinología Facultad de Medicina Pontificia Universidad Católica de Chile. Santiago, Chile.

Correspondencia a: Dr. Eugenio Arteaga eugenioarteaga@gmail.com

\section{Menopause and cardiovascular risk}

\section{L}

a enfermedad cardiovascular es la principal causa de muerte en mujeres mayores de 50 años. Previo la menopausia, el riesgo cardiovascular (RCV) es muy inferior a la de los hombres; después de ella, el riesgo aumenta aparentemente relacionado a la deficiencia estrogénica que se asocia a mayor prevalencia múltiples factores de RCV (diabetes mellitus, dislipidemia, síndrome metabólico, cambios desfavorables en el peso y distribución de grasa corporal, sensibilidad insulínica y tono simpático, entre otros $)^{1}$. De hecho, el National Cholesterol Education Program (NCEP) reconoció a la postmenopausia como un factor de RCV, asignándole el mismo peso que pertenecer al sexo masculino ${ }^{2}$. La pregunta que subsiste hasta ahora es si la Terapia Hormonal de la Menopausia (THM) es capaz de revertir el aumento de riesgo cardiovascular.

Alrededor de 40 estudios observacionales de cohorte o de casos y controles han demostrado en forma consistente que la THM reduce en $30-50 \%$ la incidencia de enfermedad coronaria y la mortalidad cardiovascular en la postmenopausia, especialmente si se administra a mujeres más jóvenes ${ }^{3}$. Los mecanismos potencialmente protectores de los estrógenos serían múltiples: vasodilatación mediada por óxido nítrico, inhibición del vasoespasmo coronario, aumento del flujo y menor resistencia vascular, aumento del débito cardíaco, facilitación de la angiogénesis y efecto antiapoptótico en los cardiomiocitos ${ }^{4}$. Otros mecanismos protectores corresponden a acciones antioxidantes y antiinflamatorias, cambios beneficios en el perfil lipídico, aumento de la sensibilidad a la insulina, atenuación del alza ponderal propia de la menopausia y menor adiposidad abdominal. Se cuentan entre los factores negativos de los estrógenos: el aumento de triglicéridos, PCR y factores de coagulación, exclusivos de la vía oral de administración de THM, y que se explica por el fenómeno denominado de "primera pasada hepática".
Sin embargo, el estudio randomizado the Women's Health Initiative (WHI) (alrededor de 27.000 mujeres de edad promedio de 63 años), vino a desafiar el concepto de cardioprotección de la $\mathrm{THM}^{5,6}$. Debe destacarse que el riesgo cardiovascular en el estudio WHI (enfermedad coronaria, accidente cerebrovascular y tromboembolismo venoso) solo se incrementó en la rama combinada con estrógenos y progestinas orales ${ }^{5}$, pero, a excepción del riesgo cerebrovascular, no se presentó en el grupo que utilizó estrógenos solos en mujeres histerectomizadas ${ }^{6}$, lo que pone en evidencia el potencial efecto negativo de la progestina utilizada, la medroxiprogesterona. Los resultados del estudio WHI, sin embargo, han sido muy criticados por defectos metodológicos (edad promedio de 63 años, morbilidades asociadas, esquema de administración y dosificación inapropiadas, etc). Un análisis posterior demostró que en el caso de la terapia combinada el aumento de riesgo de enfermedad coronaria se hacía significativo solo en el grupo etario de 70-79 años (RR 1,49 [IC 95\% $1,04-2,11])$ y no en las mujeres de menor edad ${ }^{7}$. Este fenómeno de dependencia de la edad se ha denominado "hipótesis del tiempo de uso", que postula que en mujeres más jóvenes, en general menores de 60 años, con un sistema arterial más sano, la THM favorecería la cardioprotección por los múltiples mecanismos enunciados; el inicio de THM en mujeres mayores de 60 años o con más de 10 años de postmenopausia, que pueden tener placas ateroscleróticas pre-existentes, puede asociarse a aumento de la expresión de metaloproteinasas inducido por los estrógenos causando la disrupción de la capa fibrosa y ruptura de la placa; además, el efecto pro-trombótico, exclusivo de la vía oral de administración de los estrógenos, podría favorecer fenómenos de trombosis arterial y venosa en este grupo etario.

En conclusión, la THM podría asociarse a aumento del riesgo cardiovascular en mujeres que la 
inician después de los 60 años, y podría ser neutra o protectora en las mujeres más jóvenes.

\section{Referencias}

1. Colditz GA, Willett WC, Stampfer MJ, Rosner B, Speizer $\mathrm{FE}$, Hennekens $\mathrm{CH}$. Menopause and the risk of coronaryheartdisease in women. N Engl J Med 1987; 316; 1105-10.

2. Grundy SM. Guidelines for cholesterol management: recommendations of the National Cholesterol Education Program's Adult Treatment Panel II. Heart Dis Stroke 1994; 3 (3): 123-7.

3. Hodis HN, Mack WJ. Postmenopausal hormone therapy and cardiovascular disease in perspective. Clin Obstet Gynecol 2008; 51: 546-58.

4. Salerni S, Francescomarino SQ, Cadeddu C, Acqui- stapace F, Maffei S, Callina S. The different role of sex hormones on female cardiovascular physiology and function: not only estrogens. Eur J Clin Invest 2015; 45 (6): 634-45.

5. Writing Group fort he Women's Health Initiative Investigators. Risks and benefits of estrogen plus progestin in healthy postmenopausal women: principal results from the Women's Health Initiative randomized trial. JAMA 2002; 288: 321-33.

6. The Women's Health Initiative Steering Committee. Effects of conjugated equine estrogens in postmenopausal women with histerectomy. The Women's Health Initiative randomized controlled trial. JAMA 2004; 291 : 1701-12.

7. Rossow JE, Prentice RL, Manson JE, Wu LL, Barad D, Barnabei VM, et al. Postmenopausal hormone therapy and risk of cardiovascular disease by age and years since menopause. JAMA 2007; 297 (13): 1465-77. 